\title{
Validity of Ultrasonography for the Estimation of Macrosomia among Pregnant women with Diabetes Mellitus
}

\author{
Shamsun Nahar ${ }^{1}$, Asma Khan ${ }^{2}$, TA Chowdhury³, AS Mohiuddin ${ }^{4}$ \\ ${ }^{1}$ Junior Consultant (Gynaecology \& Obstetrics), Upazila Health Complex, Sonaimuri, Noakhali, Bangladesh; ${ }^{2}$ Assistant \\ Professor, Department of Pharmacology and Therapeutics, Shaheed Suhrawardy Medical College, Dhaka, Bangladesh; \\ ${ }^{3}$ Professor, Department of Gynaecology \& Obstetrics, Bangladesh Institute of Research and Rehabilitation for \\ Diabetes, Endocrine and Metabolic Disorders, Dhaka, Bangladesh; ${ }^{4}$ Professor \& Head, Department of \\ Radiology \& Imaging, Bangladesh Institute of Research and Rehabilitation for Diabetes, \\ Endocrine and Metabolic Disorders, Dhaka, Bangladesh
}

[Received: 21 January 2017; Revised: 6 March 2017; Accepted: 11 December 2017; Published: 1 January 2018]

\begin{abstract}
Background: Estimation of fetal weight by ultrasonography is crucial for the determination of macrosomia cases among the pregnant women with diabetes mellitus. Objectives: The purpose for the present study was to validate the ultrasonography for the estimation of fetal weight among the macrosomia cases in pregnant women with diabetes mellitus. Methodology: This cross-sectional study was carried out in inpatient Department of Obstetrics and Gynecology and in outpatients Department of Radiology and Imaging, Bangladesh Institute of Research and Rehabilitation for Diabetes, Endocrine and Metabolic Disorders (BIRDEM) at Dhaka, Bangladesh during the period of April 2005 to March 2007. Pregnant women with diagnosed DM or GDM selected for caesarean section or induction of labour, gestational age 236 weeks having 23700 gm by clinical method were included in this study. First clinical estimation of fetal weight was done by the investigator then Radiologist estimated the fetal weight without knowing the EFW by clinical method. The actual birth weight was estimated after the birth of the babies. Result: It was found that in $56.5 \%$ and $60.9 \%$ cases was evaluated as macrosomia in clinical and USG examination respectively $(\mathrm{p}>0.05)$. Clinically and actually macrosomia were found $56.5 \%$ and $63.8 \%$ respectively ( $>0.05$ ). It was found that in $60.9 \%$ cases macrosomia at USG diagnosis and $63.8 \%$ case was macrosomia actually ( $>0.05)$. It was observed that out of 39 macrosomia cases evaluated clinically could ultimately evaluate $27(69.2 \%)$ cases after birth, USG detected 42 macrosomia which ultimately evaluate $30(71.4 \%)$ cases, and actually after birth macrosomia found 44(63.8\%). The validity of clinical and sonographic method for evaluation of macrosomia were studied by sensitivity, specificity and accuracy which were $61.4 \%, 52.0 \%, 58.0 \%$ and $68.2 \%, 52.0 \%, 62.3 \%$ respectively. Conclusion: In conclusion the ultrasonography is poor diagnostic tools for the estimation of macrosomia among the diabetic mother. [Journal of National Institute of Neurosciences Bangladesh, 2018;4(1): 40-44]
\end{abstract}

Keywords: Cardiology; referral; hospitalized patients

Correspondence: Dr. Shamsun Nahar, Junior Consultant (Gynaecology \& Obstetrics), Upazila Health Complex, Sonaimuri, Noakhali, Bangladesh; Email: drsweety.nahar@gmail.com; Cell no.: +8801775339803

Conflict of interest: There is no conflict of interest relevant to this paper to disclose.

Funding agency: This research project was not funded by any group or any institution.

Contribution to authors: Nahar S, Khan A, Chowdhury TA, AS Mohiuddin AS. contributed from the protocol preparation up to report writing. Nahar S, Khan A involved in the manuscript writing and revised the paper.

How to cite this article: Nahar S, Khan A, Chowdhury TA, AS Mohiuddin AS. Validity of Ultrasonography for the Estimation of Macrosomia among Pregnant women with Diabetes Mellitus. J Natl Inst Neurosci Bangladesh, 2018;4(1): 40-44

Copyright: (C2018. Nahar et al. Published by Journal of National Institute of Neurosciences Bangladesh. This article is published under the Creative Commons CC BY-NC License (https://creativecommons.org/licenses/by-nc/4.0/). This license permits use, distribution and reproduction in any medium, provided the original work is properly cited, and is not used for commercial purposes.

\section{Introduction}

Neonates whose mothers have higher glucose levels over a longer duration of pregnancy have higher incidences of macrosomia, hypoglycemia and hypocalcaemia ${ }^{1}$. Macrosomic babies have increasing intolerance to intrauterine compromise as well as an enhanced rate of birth trauma ${ }^{2}$. Best and pressman ${ }^{3}$ stated that macrosomia occurs in 25 to $42.0 \%$ of diabetic pregnancies. Macrosomia is generally used to refer to fetuses with an 
estimated fetal weight greater than or equal to $4000 \mathrm{gm}^{4}$. Macrosomia is clinically significant, as it is associated with significant neonatal and maternal morbidities, particularly in diabetic pregnancies ${ }^{5}$. The most feared result of macrosomia in a newborn is shoulder dystocia and birth asphyxia. The mothers is also 'at increased risk for Caesarean section and post partum haemorrhage. Vaginal delivery of a macrosomic infant increases the risk of third and fourth degree lacerations fivefold ${ }^{6}$.

By clinical method of foetal weight estimation, the volume of amniotic fluid, the size and configuration of the uterus and maternal body habitus complicate estimation of the size of the fetus by palpation through the abdominal wall. Several studies have documented mean errors of about $300 \mathrm{gm}^{7}$. By ultrasonogrpahic estimation of foetal weight, the typical mean error ranges from 300 to $550 \mathrm{gm}$. A study comparing fetal weight estimates of clinicians, multiparous patients and ultrasonography found that the ultrasound was the least accurate of the three methods. Limitations in the sensitivity and specificity of ultrasound have been observed in other studies. Despite these limitations, clinicians continue to incorrectly believe that ultrasound is an accurate way of predicting macrosomia?

In the above context the present work has been designed to validate the ultrasonography for the estimation of fetal weight among the macrosomia cases in pregnant women with diabetes mellitus.

\section{Methodology}

This was a prospective, consecutive, non-interventional and non-randomized cross sectional study. The study was carried out in the department of Obstetrics and Gynecology, BIRDEM in (collaboration with the department of Radiology and Imaging department of the same institute. The study was carried out for a period of two years from April 2005 to March 2007. Prior to the commencement of this study, the research protocol was approved by the Local Ethical Committee of BIRDEM Academy. Pregnant Women with pregestational and gestational diabetes mellitus having fasting blood sugar level $26.1 \mathrm{mmol} / 1$ aged from 20-40 years and gestational age 36 Weeks admitted in inpatient Department of Obstetrics and Gynecology, BIRDEM and attending in outpatient Department of Radiology and Imaging of the same institute are selected as subjects. Non-randomized consecutive sampling was used to collect the data. Pregnancy of 36 weeks with diagnosed pregestational DM and GDM having fasting blood sugar level $26.1 \mathrm{mmol} / \mathrm{L}$ (as per WHO Expert Committee 1999) selected for caesarean section or induction of labour, accurate gestational age regular menstrual cycle with exact last menstrual period and having early ultrasonography, longitudinal lie, cephalic presentation, intact membranes and estimated fetal weight $23700 \mathrm{gm}$ by clinical method were included as study population. Pregnancy less than 36 weeks, pregnancy with pregestational DM or GDM with complication (e.g. hypertension, ketoacidosis etc), presence of uterine tumour, ruptured membranes, malpresentation, multiple pregnancy, diagnosed fetal anomaly, excessive obesity of the mothers Where symphysio-fundal height can not be easily measured and estimated fetal weight $<3700$ gm by clinical method were excluded from this study. Those who were agreed to take part in this study were selected. Written consent was taken from the patients. Then detailed history was taken and clinical examination was done and those who were clinically macrosomic were sent for ultrasonographic estimation of the fetal weight. Once the babies were born, their actual birth weights were measured by weight machine. All the information were recorded in a pre-designed data collection sheet. Clinical estimation of fetal weight was estimated by the investigator. Patient preparation: Before estimation of fetal weight by clinical method bladder was evacuated. Positioning of the patient and measurement: patient was placed in supine position and abdomen was exposed as necessary and then uterus was placed in midline position and then symphysio fundal height was taken by non elastic tape with $\mathrm{cm}$ with the side of the tape facing downwards provided the uterus was relaxed. Then fetal weight was estimated (lohnson, 1957; McCormick, 2000). Ultrasonographic estimation of fetal weight was done by a Radiologist without knowing the estimated fetal weight by clinical method. Estimation of actual birth weight was estimated by calibrated weight machine. The data sheets were $100 \%$ scrutinized to check the quality of the raw data. The hundred percent cross check were done after editing. Collected data were entered into the computer and processed by it. Data were analyzed by, software SPSS (Statistical Package for Social Science). Paired ' $t$ ' test and chi square test and $\mathrm{Z}$ test were used Where it Was applicable. The results were presented in Tables and Figures and were expressed as mean i Standard Deviation. For the validity of the study outcome, sensitivity, specificity and accuracy of clinical estimation of fetal Weight and sonographic estimation of fetal Weight Were calculated. Difference was considered statistically significant if $\mathrm{p}$ value was $<0.05$.

\section{Results}

A total number of 69 pregnant women were recruited 
for this study. The mean age of the study subjects was 30.8 years with standard deviation 15.1 years and ranged from 20 to 40 years. The maximum pregnant woman was found between 26 to 30 years age range and minimum was found between 36 to 40 years age range (Tab1e- Iand figure 1).

Table 1: Age distribution of the study subjects $(\mathrm{n}=69)$

\begin{tabular}{lcc}
\hline Age Group & Frequency & Percent \\
\hline 20 to 25 Years & 27 & 39.1 \\
26 to 30 Years & 30 & 43.5 \\
31 to 35 Years & 8 & 11.6 \\
36 to 40 Years & 4 & 5.8 \\
Total & $\mathbf{6 9}$ & $\mathbf{1 0 0 . 0}$ \\
Mean +SD & $30.8+15.1$ (years) & \\
\hline
\end{tabular}

The pregnant women having macrosomia evaluated clinically were associated with the actual birth weight. Gut of 69 cases; 44 cases had findings of macrosomia and 25 cases were negative for macrosomia (normal) in actual birth weight. Whereas clinically evaluated 39 cases as macrosomia and 30 cases as normal. Out of 39 macrosomic cases evaluated clinically 27 cases were actually macrosomic and 12 cases were normal after birth. Out of 30 normalcases evaluated clinically 17 cases were macrosomic and 13 cases were actually normal. The difference between clinically evaluated macrosomia and actual macrosomia, between clinically evaluated normal and actually normal cases was not statistically significant $(\mathrm{p}>0.05)$ (Table 2$)$.

Table 2: Association between clinical diagnosis of macrosomia and actual birth weight $(n=69)$

\begin{tabular}{lccc}
\hline Clinically & \multicolumn{2}{c}{ Actual Macrosmia } & Total \\
\cline { 2 - 3 } Macrosmia & Present & Absent & \\
\hline Present & 27 & 12 & 39 \\
Absent & 17 & 13 & 30 \\
Total & $\mathbf{4 4}$ & $\mathbf{2 5}$ & $\mathbf{6 9}$ \\
\hline
\end{tabular}

Chi square $=1.16, \mathrm{df}=1, \mathrm{p}=0.281, \mathrm{NS}=$ Not significant

The pregnant women having macrosomia diagnosed sonographicaily were associated with the actual birth weight. Out of 69 cases; 44 cases had findings of macrosomia and 25 .cases were negative for macrosomia (normal) in actual birth weight. Whereas in USG findings detected 42 cases as macrosomia and 27 cases as normal. Out of these 42 macrosomic cases detected sonogrophically 30 cases were actually macrosomia and 12 cases were normal after birth. Out of 27 normal cases detected sonographically 14 cases were macrosomic and 13 cases were actually normal.
The difference between sonographically detected macrosomia and actual macrosomia, between sonographically detected normal and actual normal cases was not statistically significant $(\mathrm{p}>0.05)$ (Table $3)$.

Table 3: Association between USG diagnosis of macrosomia and actual birth weight $(n=69)$

\begin{tabular}{lccc}
\hline USG & \multicolumn{2}{c}{ Actual Macrosmia } & Total \\
\cline { 2 - 3 } Macrosmia & Present & Absent & \\
\hline Present & 30 & 12 & 42 \\
Absent & 14 & 13 & 27 \\
Total & $\mathbf{4 4}$ & $\mathbf{2 5}$ & $\mathbf{6 9}$ \\
\hline
\end{tabular}

Chi square $=2.73, \mathrm{df}=1, \mathrm{p}=0.098$

The sensitivity, specificity and accuracy of clinical and sonogrphic evaluation of macrosomia were $61.4 \%$, $52.0 \%$ \&: $58.0 \%$ and $68.2 \%, 52.0 \%$ \& $62.3 \%$ respectively (Table 4).

Table 4: Sensitivity, specificity and accuracy of clinical and ultrasonography in evaluation of macrosomia

\begin{tabular}{lcc}
\hline Validity test & Clinical & Sonography \\
\hline Sensitivity & $61.4 \%$ & $68.2 \%$ \\
Specificity & $52.0 \%$ & $58.0 \%$ \\
Accuracy & $52.0 \%$ & $62.3 \%$ \\
\hline
\end{tabular}

\section{Discussion}

Evaluating fetal weight is an important part of obstetrics $^{8}$. Accurate estimation can help in deciding the timing and mode of delivery of macrosomic fetuses. An accurate diagnosis of macrosomia can lead to a decrease in perinatal morbidity. Its prediction may enable the physician and staff to prepare for shoulder dystocia or prevent a traumatic injury. Methods of evaluating fetal weight include 1 clinical and ultrasonographic. Since ultrasonographic facilities are expensive, not easily available and trained personnel are required, it would be immensely useful to know if other simpler clinical 'methods can estimate fetal weight with the same degree of accuracy ${ }^{8}$.

Numerous studies have challenged the accuracy of sonographic birth Weight estimation and have concluded that sonography may be no more accurate for the prediction of birth weight than clinical palpation. Untrasonography is not available in very remote areas of Bangladesh. Even when available, not all patients can afford the cost. Many of the rural obstetric population in this country are not sure of their dates. So, gestational age derived birth weight centiles are also unsuitable for those women with unsure dates who cannot afford or access ultrasonography? 
Macrosomia is generally used to refer to fetuses with an estimated fetal weight greater than or equal to 4000 gram (Rahimian and Varner, 2003). Nahum (2000) with his colleagues developedan equation for predicting fetal macrosomia based on maternal demographic and pregnancy-specific factors alone and by using this equation, they predicted term birth within i7.6\% (267g) and they choose a cut off value of $3,775 \mathrm{~g}$ for prediction of fetal macrosomia.

Several studies have documented mean errors of estimation of fetal weight by clinical method is about $3000 \mathrm{gm}^{4}$. In the above context, this study included clinically estimated fetal weight $3000 \mathrm{gm}$ as inclusion criteria for macrosomia though macrosomia actually means $2.4 \mathrm{~kg}$.

The present study findings were discussed and compared with previously published relevant studies. Macrosomia is generally used to refer to fetuses with an estimated fetal weight greater than or equal to 4000 gram $^{4}$. Nahum ${ }^{6}$ with his colleagues developed an equation for predicting fetal macrosomia based on maternal demographic and pregnancy-specific factors alone. In the present study it was observed that there was no significant difference between clinical, ultrasonographic estimation of fetal weight and actual birth weight. Banerjee et $\mathrm{al}^{8}$ have also made almost identical observations. According to that study clinical method of evaluating fetal weight is as good as ultrasonographic estimation.

The difference between clinical evaluation of macrosomia and actual macrosomia and between sonographic evaluation of macrosomia and actual macrosomia was not statistically significant. Watson et a ${ }^{10}$ noted that both clinical and sonographic methods of predicting birth weight had a similar accuracy even among macrosomic fetuses. Chauhan et $\mathrm{al}^{11}$ shows sonographic models were not significantly superior to clinical examination in detecting newborns with birth-weights 24,000 g. All these results support the present study.

Iohnstone et $\mathrm{al}^{12}$ observed clinical examination is as predictive as ultrasound measurements but at the sometime also observed that no matter how data were presented they show that clinical and ultrasound measurements are poor predictors of macrosomia. In the present study, it was observed that sensitivity, specificity and accuracy of clinical evaluation of macrosomia were $61.4 \%, 52.0 \%$ and $58.0 \%$ respectively and of ultrasonographic evaluation of macrosomia were $68.2 \%, 52.0 \%$ and $62.3 \%$ respectively.
Nahum $^{6}$ studied different techniques like clinical estimation, patients self estimation, sonographic estimation and maternal characteristics for predicting term fetal macrosomia. The sensitivity and specificity of clinical and onographic estimation of that study was $54.0 \% \& 95.0 \%$ and $59.0 \% \& 90.0 \%$ respectively. The findings are in agreement with the present study. Noumi et a ${ }^{13}$ have shown that coefficient of correlation between the clinical and sonographic EFW and the actual birth weight were $0.62(\mathrm{p}<0.001)$ and 0.66 $(p<0.001)$ respectively. The sensitivity, specificity, positive and negative predictive values of predicting macrosomia by both modalities were $50.0 \%, 95.0 \%$, $.0 \%, 97.0 \%$ and $50.0 \%, 97.0 \%, 50.0 \%$ and $97.0 \%$ respectively. The results of the present study agree with these investigations.

\section{Conclusion}

There is no significant difference between clinical and ultrasonographic methods of estimation of macrosomia. So, either clinical or ultrasonographic method of estimation may be considered to estimate fetal macrosomia. As the study was conducted with a small number of subjects, further study may be undertaken in future with large number of subjects.

\section{References}

1. Metzger BE, Buchanan TA, Coustan DR, De Leiva A, Dunger DB, Hadden DR, Hod M, Kitzmiller JL, Kjos SL, Oats JN, Pettitt DJ. Summary and recommendations of the fifth international workshop-conference on gestational diabetes mellitus. Diabetes care. 2007;30(Supplement 2):S251-60

2. Xiong X, Saunders LD, Wang FL, Demianczuk NN. Gestational diabetes mellitus: prevalence, risk factors, maternal and infant outcomes. International Journal of Gynecology \& Obstetrics. 2001;75(3):221-8.

3. Best G, Pressman EK. Ultrasonographic prediction of birth weight in diabetic pregnancies. Obstetrics \& Gynecology. 2002;99(5):740-4.

4. Rahimian I and Varnar MW. Disproportionate fetal growth. ln: DeCherney AH, Nathan L (eds), Current Obstetrics and Gynecologic Diagnosis and Treatment, 9*h edn, Mc Graw Hill, New York 2003; pp. 301-14

5. Crowther CA, Hiller JE, Moss JR, McPhee AJ, Jeffries WS, Robinson JS. Effect of treatment of gestational diabetes mellitus on pregnancy outcomes. New England Journal of Medicine. 2005;352(24):2477-86.

6. Nahum GG. Detecting and managing fetal macrosomia. Contemporary Ob Gyn. 2000;45(6):89-119

7. Zamorski MA and Biggs WS. Management of suspected fetal macrosomia. Am Fam Physician 2001;63:302-6

8. Banerjee K, Mittal S, Kumar S. Clinical vs. Ultrasound evaluation of fetal weight. Int J Gynecol Obstet 2004;86:41-43

9. Haque FMA. Comparative study between clinical and sonographic estimation of fetal weight in third trimester of pregnancy. Dissertation 2005; Bangladesh College of Physicians and Surgeons. 
10. Watson WJ, Soisson AP, Harlass FE. Estimated weight of the term fetus. Accuracy of ultrasound vs. clinical examination. The Journal of reproductive medicine. 1988 Apr;33(4):369-71.

11. Chauhan SP, Lutton TC, Bailey KI, Morrison IC. Lntrapartum prediction of birth weight: clinical versus sonographic estimation based on femur length alone. Obstet Gynecol 1993;81:695
12. Iohnstone FD, Prescott RI, Steel IM, Mao IH, 'Chambers S, Muir N. Clinical and ultrasound prediction of macrosomia in diabetic pregnancy. Br I Obstet Gynaecol 1996;103:747-53

13. Noumi G, Collado-Khoury F, Bombard A, Iulliard K, Weiner Z. Clinical and sonographic estimation of fetal weight performed during labour by resident. Am I Obstet Gynecol 2005;192:1407-9 